\title{
EL PROBLEMA DEL REPARTO EQUITATIVO EN LA CONVEMAR: ALGUNOS ARGUMENTOS DE JUSTICIA EN FAVOR DE LOS ESTADOS EN DESARROLLO SIN LITORAL
}

LOSC's challenge of equal sharing: some justice based arguments in favor of landlocked developing countries

\author{
NiCOLE SelAmÉ GLENA* \\ Universidad de Valparaíso
}

\begin{abstract}
RESUMEN
La CONVEMAR modificó la distribución de los espacios marítimos. La ZEE expandió los derechos soberanos de los Estados ribereños, sin considerar a los Estados sin litoral, mientras que la Zona se constituyó como un espacio libre de soberanía estatal cuya explotación debe ir en beneficio de la humanidad. Esto supone un reparto equitativo de los beneficios entre todos los Estados miembros. La CONVEMAR, no obstante, no incluye mecanismos para determinar la distribución 'equitativa'. En este trabajo propongo una interpretación basada en la equidad que compense a los Estados en desarrollo sin litoral por su exclusión en la ZEE, y sus necesidades de desarrollo sostenible insatisfechas. Para ello, utilizo argumentos de responsabilidad colectiva, justicia compensatoria y suficientarismo.
\end{abstract}

\section{PALABRAS CLAVE}

Patrimonio común de la humanidad, Estados sin litoral, reparto equitativo.

\section{ABSTRACT}

UNCLOS modified the distribution of maritime spaces. The EEZ expanded the sovereign rights of coastal States, without considering the landlocked States, while the Area was established as a space free of state sovereignty whose exploitation must be for the benefit of mankind. This supposes an equitable distribution of the benefits between all member States. UNCLOS, however, does not include mechanisms to determine an 'equitable' distribution. In this paper, I propose an interpretation based on equity that compensates landlocked developing States for their exclusion in the EEZ, and their unsatisfied sustainable development needs. For this, I use arguments of collective responsibility, compensatory justice and sufficientarianism.

KEYWORDS

Common heritage of mankind, landlocked states, equal share.

\section{Introducción}

La Convención sobre Derecho del Mar (CONVEMAR) de 1982 modificó la relación de los Estados con el mar. La nueva división del territorio marítimo tuvo efectos distributivos importantes. Por una parte, creó la Zona Económica Exclusiva (ZEE), lo que significó una expansión sin precedentes de los derechos soberanos y exclusivos de los Estados ribereños hasta 200 millas marinas desde las líneas de base, repartición de la que quedaron excluidos los Estados sin litoral. Por otra parte, la CONVEMAR creó la Zona más allá de la jurisdicción nacional (la Zona) y la declaró patrimonio común de la humanidad ( $\mathrm{PCH})$, constituyéndola como un área libre de soberanía estatal, pacífica, gobernada en conjunto por las naciones, que debe ser preservada

\footnotetext{
* Universidad de Valparaíso, Valparaíso, Chile. Correo electrónico: nicole.selame@uv.cl. Este trabajo fue desarrollado en el marco del proyecto FONDECYT de iniciación 11180038.
} 
para las futuras generaciones y cuyos beneficios deben distribuirse de manera equitativa entre toda la humanidad.

En este trabajo exploro el principio de equidad incluido en la regulación de la Zona, sosteniendo que una interpretación distinta de la aritmética es la más apropiada por razones históricas y de justicia. Señalo que para que la explotación de la Zona vaya en interés de la humanidad, es necesario que exista una distribución diferenciada de los beneficios, y justifico que una distribución equitativa incluye un trato preferente a los Estados en desarrollo sin litoral, basada en la necesidad de compensar a estos Estados por su exclusión en la ZEE. Esta interpretación no desconoce que puedan existir otros argumentos en favor de otros Estados. Si bien esta exclusión operó respecto de todos los Estados sin litoral -desarrollados y en desarrollo, me concentro únicamente en los Estados sin litoral en desarrollo, pues respecto de ellos operan argumentos adicionales de justicia relacionados con la responsabilidad colectiva y principios suficientarios de justicia ${ }^{1}$.

El artículo se desarrolla de la siguiente manera. En la segunda sección explico la forma en que la CONVEMAR dividió el territorio marítimo y los efectos distributivos que produjo (2.1). Además, doy cuenta de cómo parte importante de la Convención se vio modificada por medio del Acuerdo de Implementación de 1994, alterando las normas que favorecían a los Estados en desarrollo (2.2). La tercera sección presenta las características especiales de los Estados en desarrollo sin litoral (3.1), su regulación en el Derecho internacional (3.2) y en la CONVEMAR (3.3). Por último, en la cuarta sección exploro el principio de PCH (4.1) y el problema del reparto equitativo de beneficios (4.2), analizando el principio de equidad a la luz de principios suficientarios de justicia, y argumentos de justicia compensatoria y de responsabilidad colectiva (4.3 y 4.4). Concluyo en la quinta sección (5) que es posible interpretar el reparto equitativo de manera que dé preferencia a los Estados en desarrollo sin litoral, sin perjuicio del tratamiento favorable que pudiera caberle a otros Estados en desarrollo.

\section{La Convención sobre Derecho del Mar de 1982}

\subsection{La división de los espacios marítimos y sus efectos distributivos}

La CONVEMAR estableció un orden legal para los mares con el objeto de lograr la libre comunicación, la paz, el acceso igualitario a los recursos, la conservación de los recursos vivos y la preservación del medio marino (preámb.). Para ello, dividió el océano en áreas, fijando sus límites y estableciendo los derechos y obligaciones que corresponden a cada Estado. La parte II regula el mar territorial y la zona contigua, la parte V la ZEE, la VI la plataforma continental, la VII la alta mar y la parte XI la Zona. En este trabajo, me concentro en la regulación de la ZEE y la Zona, poniendo especial atención sus efectos distributivos.

La ZEE es una innovación introducida por la Convención que soluciona conflictos entre los Estados por el control de los recursos, al crear y repartir derechos soberanos exclusivos sobre el territorio. Esta nueva distribución, sin embargo, generó tensiones al expandir los derechos solo de algunos Estados, los ribereños, dejando fuera a los Estados sin litoral. Con la creación de la ZEE, la Convención fijó un área de 200 millas marinas desde las líneas de base, donde los Estados ribereños tienen derechos soberanos y exclusivos de exploración, explotación, conservación y administración, entre otros (art. 55, 56 y 57). Su consecuencia directa fue una disminución considerable de la alta mar: antes de la Convención se consideraba alta mar todo aquello que quedara fuera del mar territorial y las aguas interiores, o sea, todo menos las 12 millas marinas; con su entrada en vigor, el área de soberanía exclusiva se extendió a 200 millas.

\footnotetext{
${ }^{1}$ Entenderé la responsabilidad colectiva a nivel global en dos sentidos; uno, la responsabilidad causal, esto es, aquella que se funda en el hecho de haber provocado el daño y la necesidad de repararlo y, dos, la responsabilidad remedial, que tiene lugar en los casos en que la obligación derive de la existencia de una situación manifiestamente injusta, aunque no la hayamos provocado. Al referirme al suficientarismo estaré apelando a aquellas teorías de la justicia que consideran que tenemos la obligación de poner a todas las personas sobre un determinado umbral. Si bien estos conceptos se encuentran desarrollados en la última sección del trabajo, para un mejor conocimiento de las discusiones acerca de la responsabilidad colectiva y del suficientarismo véase respectivamente MILLER (2007) y CASAL (2007).
} 
Esto no estuvo exento de detractores. Para las potencias marítimas que realizaban actividad pesquera a distancia, por ejemplo, significaba una importante disminución de los sitios de pesca. Los mayores opositores fueron los Estados sin litoral y geográficamente desaventajados, quienes quedaron excluidos de la repartición. Actuando como grupo de interés, durante las negociaciones manifestaron su expectativa de obtener una proporción mayor de los beneficios de la explotación de la Zona como una reparación por la extensión de los derechos soberanos y por su situación de desventaja respecto de los recursos marinos ${ }^{2}$. A pesar de las presiones, sin embargo, estos últimos no lograron más que derechos residuales sobre los excesos de los recursos de la ZEE de sus vecinos ${ }^{3}$.

Una segunda innovación de la CONVEMAR es la creación de la Zona, esto es, el área más allá de la jurisdicción nacional de los Estados. Esta comprende los fondos marinos y oceánicos y su subsuelo fuera de los límites de la jurisdicción nacional (art. 1). Al igual que la alta mar, su delimitación se establece negativamente, pues corresponde a aquella parte que queda más allá de la plataforma continental o de las 200 millas marinas, en caso de que la plataforma sea más pequeña. No se trata de un volumen de agua, sino del suelo y subsuelo marino, incluidos los recursos no vivos que se encuentren en él. Es, en otras palabras, el fondo marino bajo la alta mar.

A diferencia de los otros espacios marítimos, la Zona recibe una regulación especial y detallada en la CONVEMAR. Esto obedece, por una parte, a su atractivo económico, que se encuentra en los nódulos polimetálicos, sulfitos polimetálicos y corteza de ferromanganeso presentes en el suelo y subsuelo marino, y a su potencial redistributivo; y, por otra parte, a su interés medioambiental en convertirse en un área de conservación para la preservación de la biodiversidad.

Siguiendo los principios propuestos por Arvid Pardo en su discurso de $1967^{4}$ e incluidos en la resolución 2749 (XXV) de la Asamblea General de la ONU de 1970, la Convención declara la Zona y sus recursos patrimonio común de la humanidad (arts. 136 y 137), establece la obligación de que su explotación vaya en beneficio de toda la humanidad, y crea la Autoridad Internacional del Fondo Marino (AIFM) para representarla y proteger sus intereses. Con esta regulación, la Convención asumió una función redistributiva e integradora, al velar porque todos los Estados puedan participar de las actividades de exploración y explotación y de sus beneficios, y no solo aquellos que cuenten actualmente con los recursos financieros y tecnológicos.

De acuerdo con esto, todas las actividades que se realicen en la Zona deben ir en beneficio de toda la humanidad, independientemente de la ubicación geográfica de los Estados, y tomando en cuenta especialmente los intereses y necesidades de los Estados en desarrollo y de los pueblos que no hayan logrado la plena independencia u otro régimen de autonomía (art. 140). La CONVEMAR señala que debe existir respecto de estos países una consideración especial en relación con la distribución equitativa de los beneficios financieros y otros beneficios económicos derivados de las actividades en la Zona (arts. 140 y 160).

Para garantizar este principio, la parte XI de la Convención contiene una serie de normas que regulan la exclusiva utilización con fines pacíficos (art. 141), la protección del medio marino (arts. 145 y 147), la protección de la vida humana (art. 146) y la participación de los Estados en desarrollo en las actividades a realizarse en la Zona (art. 148), incluida la transferencia de tecnología (art. 144). Para fomentar la participación de los Estados en desarrollo en estas actividades se creó un sistema paralelo en que la AIFM, en representación de la humanidad, puede actuar a través de su órgano operativo - la "Empresa" - en la explotación del fondo marino. De esta forma, se esperaba que se representaran los intereses y necesidades de los Estados en desarrollo y sin autonomía ${ }^{5}$.

\footnotetext{
${ }^{2}$ TUERK (2015), p. 336.

${ }^{3}$ El artículo 69 de la CONVEMAR establece el derecho de los Estados sin litoral y geográficamente desaventajados a participar, sobre una base equitativa, en la explotación de una parte apropiada del excedente de los recursos vivos de la ZEE de los Estados de su región o subregión.

${ }^{4}$ NACIONES UNIDAS (1967).

${ }^{5}$ BOURREL et al. (2016), p. 3.
} 


\subsection{Modificación de la Parte XI, relativa a la Zona, a través del Acuerdo de Implementación de 1994}

Las normas de la Parte XI daban cuenta de la visión progresista e integradora de la Convención, que buscaba crear un orden global para los mares más justo y equitativo. Esta postura, no obstante, hizo que su vocación universal corriera peligro. La CONVEMAR se abrió para firmas en 1982 y solo pudo entrar en vigor en 1994 luego de la ratificación número 60 de Guyana. El problema era que entre las ratificaciones solo había una de un país desarrollado: Islandia ${ }^{6}$. Esto resultaba problemático. Como señala Churchill, "[u]na Convención en que solo los países en desarrollo fueran Estados parte era claramente indeseable, potencialmente divisorio, y no cumplía con las aspiraciones de la Asamblea General de participación universal"7. Además, una Convención en que no participaran los países desarrollados sería desastrosa, ya que son estos los que más usan el mar, más contaminan y participan en mayor medida de las disputas $^{8}$.

Con el objeto de lograr una ratificación masiva, la ONU realizó una consulta entre los Estados desarrollados que finalmente se materializó en el Acuerdo de Implementación de 1994 (Al). Este acuerdo buscaba facilitar la participación universal mediante la modificación de la Parte XI, tomando en cuenta los cambios políticos y económicos (preámb. 5 y 6 ) y moderando la postura que favorecía a los Estados en desarrollo. Formalmente, se trata de un acuerdo para una mejor aplicación de la CONVEMAR. Sin embargo, los Estados desarrollados usaron este instrumento para modificar o dejar sin efecto las normas que les resultaban problemáticas. Se trata de una modificación por acuerdo subsecuente y parte de la doctrina ha sostenido que la práctica sobreviniente ha llevado a la adherencia tácita de todas las partes de la CONVEMAR ${ }^{9}$. En este sentido, el Al se entiende incorporado a la Convención y, en caso de discordancia, este se aplica de manera preferente (art. 2). Si bien el Al no alteró los propósitos de la CONVEMAR ni el $\mathrm{PCH}^{10}$, sí produjo cambios importantes en la estructura distributiva de la misma, en la medida que modificó sustancialmente la Parte XI sobre la Zona, en que los Estados desarrollados tenían un fuerte interés económico.

Como señalé, la CONVEMAR originalmente contemplaba normas que directamente beneficiaban a los Estados no desarrollados, buscando incorporarlos en las actividades en la Zona y teniendo en cuenta especialmente sus intereses y necesidades a la hora de distribuir los beneficios. Estas normas, incluidas al alero del Nuevo Orden Económico Internacional (NOEI), quedaron mayoritariamente sin efecto con el Al. Los Estados desarrollados justificaron la reconsideración de la Parte XI apelando a los cambios políticos y económicos (clausula rebus sic stantibus), especialmente la confianza creciente en los principios de mercado (preámb. Al). A nivel interno, los Estados desarrollados estaban reduciendo el sector público por medio de privatizaciones, y esperaban que estos principios se trasladaran al ámbito internacional ${ }^{11}$.

Los Estados industrializados finalmente impusieron su visión capitalista y de mercado ${ }^{12}$, condicionando su firma a modificaciones medulares al régimen de la Zona. Una buena manera de ilustrar los cambios introducidos por el $\mathrm{Al}$ al régimen de la Zona son las modificaciones que sufrió la Empresa, el organismo de la AIFM encargado de desarrollar la minería submarina en representación de los Estados en desarrollo. Primero, el Al eliminó la obligación de los Estados parte de financiarla. Esto significa que ahora la Empresa se ve obligada a participar de la minería submarina únicamente a través de joint ventures (empresas mixtas). Junto con ello, condicionó la posibilidad de participación de la Empresa a una decisión del Consejo de la AIFM, para lo que

\footnotetext{
${ }^{6}$ ANDERSON (1995), p. 275.

${ }^{7}$ CHURCHILL (2015), p. 26.

8 LODGE (2015), p. 227.

${ }^{9}$ BUGA (2015), p. 62.

${ }^{10}$ BUGA (2015), p. 53.

${ }^{11}$ ANDERSON (1995), pp. 275 y 276.

${ }^{12}$ ANDERSON (1995), pp. 275 y 276.
} 
debe presentarse un plan de explotación por parte de cualquier entidad calificada o una postulación para un joint venture ${ }^{13}$. Esto limitó la autonomía de la Empresa ${ }^{14}$.

Otro ejemplo lo constituye la alteración del sistema para la transferencia de tecnología, fundamental para habilitar la participación de los Estados en desarrollo en la exploración y explotación de la Zona. Si antes la CONVEMAR planteaba dicha transferencia como una obligación de los Estados desarrollados para beneficio de los en desarrollo, ahora el Al señala que debe ajustarse a las reglas de mercado (art. 5, Anexo III). Por último, la regulación de la explotación comercial fue modificada para dejar sin efecto las limitaciones a la producción, alegando que podían actuar como un desincentivo a la inversión. El Al enfatiza que la explotación de los recursos en la Zona debe hacerse en consonancia con principios comerciales, sin subsidio a las actividades y equiparando la minería submarina a la terrestre ${ }^{15}$.

De esta forma, el Al alteró drásticamente el balance entre los intereses de los Estados desarrollados y los no desarrollados, al disminuir la posibilidad de que estos últimos participen en las actividades de explotación de la Zona. A pesar estas modificaciones, el principio PCH no fue modificado; el propio preámbulo del Al se refiere expresamente a que la Zona y sus recursos son patrimonio común de la humanidad. Como señala Anderson, el PCH constituye parte fundamental del equilibrio alcanzado entre los países desarrollados y en desarrollo ${ }^{16}$. Aunque se haya realizado una alteración pro-mercado de la CONVEMAR para lograr la ratificación de los Estados desarrollados se mantuvo el principio de PCH así como la necesidad de lograr un reparto equitativo de los beneficios derivados de la minería submarina, tomando especialmente en cuenta los intereses y necesidades de los Estados en desarrollo, sean ribereños o sin litoral.

\section{Los Estados sin litoral y la CONVEMAR}

\subsection{Los Estados sin litoral}

El artículo 124 de la CONVEMAR define los Estados sin litoral como aquellos que no tienen costa marítima. Constituyen hoy casi la cuarta parte de los Estados del mundo y se encuentran repartidos en África (16), Asia (12), Europa (14) y Sudamérica (2). De ellos, solo 12 son desarrollados, mientras que otros 15 son considerados Estados en desarrollo y 17 Estados menos desarrollados ${ }^{17}$. En general, los Estados sin litoral tienen un bajo desempeño en el Índice de Desarrollo Humano. Las personas en estos países viven menos años, tienen peor educación y un ingreso per cápita más bajo que si vivieran en un país costero ${ }^{18}$. Muchos Estados sin litoral se encuentran en situación de precariedad: 13 de ellos tienen un producto interno bruto per cápita inferior a $\$ 1.000$ dólares y más del $30 \%$ de la población total vive bajo la línea de la pobreza establecida por el Banco Mundial. Al menos 150 millones de personas deben vivir con menos de dos dólares al día ${ }^{19}$.

Fuera de Europa, todos los Estados sin litoral se ven enfrentados a grandes dificultades ${ }^{20}$. Por regla general, estos se encuentran en regiones pobres y extensas, y cuentan con infraestructura de transporte y sistemas logísticos deficientes, acuerdos de cooperación débiles

\footnotetext{
${ }^{13} \mathrm{El}$ 'sistema paralelo' establecido antes del Al suponía que por cada sitio cuya concesión se entregara a un Estado desarrollado para su explotación, debía dejarse un área reservada de igual valor comercial para que fuera explotada en favor de los países en desarrollo. Esto podía realizarse por la Empresa actuando sola, o en conjunto con un país en desarrollo. Con el Al se limita esta posibilidad autorizándola únicamente a actuar como empresa conjunta, lo que por las condiciones de los países en desarrollo es más difícil y menos probable.

${ }^{14}$ LODGE (2015), p. 239.

15 LODGE (2015), p. 244.

${ }^{16} \mathrm{El}$ Acuerdo de Implementación 'seems to have struck a fair balance between the principle of the Common Heritage of Mankind and the aspirations of developing countries on the one hand, and current attitudes towards economic issues and the positions of industrialised countries, on the other.' ANDERSON (2015), p. 289.

17 UN-OHRLLS (2019).

18 UNDP (2019), pp. 308-311.

19 UN-OHRLLS (2018).

${ }^{20}$ En este trabajo exluiré a los Estados sin litoral europeos, quienes por las particulares carácterísticas del continente y de la Unión Europea son los únicos desarrollados y no se ven enfrentados a las mismas dificultades que el resto. Véase CASAL Y SELAMÉ (2015), pp. $270-271$.
} 
y fronteras difíciles de transitar. La lejanía del mar, física y administrativa, hace que el transporte terrestre sea mucho más caro, lento y peligroso. Esto aumenta los precios de las importaciones y exportaciones, y amplía la probabilidad de que existan daños, pérdidas y robos ${ }^{21}$. La distancia promedio para los Estados sin litoral hasta el puerto más cercano es de 1.370 kilómetros $^{22}$; sin embargo, no siempre es la mejor opción disponible, y muchas veces deben tomar rutas más largas atendiendo a otros factores como la falta de infraestructura, conflictos armados o problemas con los países de tránsito.

A diferencia de las regiones interiores de países de gran superficie, la distancia no es el único factor que los afecta. Los países sin litoral además cuentan con el problema adicional de quedar entregados a la voluntad de sus vecinos para poder llegar al mar. En algunos casos, como Bolivia o Nepal, la distancia es mínima pero las dificultades asociadas con el cruce de fronteras y el tránsito por el territorio extranjero son enormes. Los Estados sin litoral no solo dependen de que los dejen cruzar de un Estado al otro, sino que además quedan sometidos a la logística e infraestructura del país de tránsito, su sistema aduanero y tributario y sus condiciones de paz y estabilidad $^{23}$. Esto hace mucho más difícil que puedan salir al mar y comerciar, sobre todo considerando que los vecinos tienen a su vez bajo desarrollo y muchas veces compiten en el mercado con los mismos productos.

\subsection{Regulación de los Estados sin litoral en el derecho internacional}

Existe creciente preocupación respecto de la situación desventajosa de los Estados sin litoral en el ámbito internacional. Esta tiene que ver principalmente con las dificultades estructurales que enfrentan para crecer de manera sostenible. Sin contar con una economía diversificada ni recursos tecnológicos, no tienen otra alternativa que hacer un uso intensivo y no sostenible de sus recursos naturales. Así, los Estados en desarrollo sin litoral sufren un doble problema. Por una parte, no pueden acceder a medios alternativos de desarrollo menos contaminantes y distintos de las actividades extractivistas. Por la otra, son más vulnerables a los problemas medioambientales, en la medida que están menos preparados para enfrentar las dificultades que se produzcan por el calentamiento global, como los cambios de temperaturas y de los niveles del mar, las alteraciones en los ciclos de producción de alimentos, la propagación de enfermedades, etc. ${ }^{24}$.

Mejorar las condiciones de estos Estados es fundamental, tanto para disminuir pobreza y la desigualdad como para la protección del medio ambiente. Para lograr estos objetivos, la respuesta más frecuente de la comunidad internacional ha sido promover acuerdos de integración y cooperación económica que buscan insertar a estos Estados en el comercio global $^{25}$. Sin embargo, esta estrategia tiene limitaciones importantes.

La ONU ha reconocido los problemas que enfrentan estos Estados para lograr los Objetivos de Desarrollo del Milenio (2000) y, más recientemente, los Objetivos de Desarrollo Sostenible $(2015)^{26}$. La comunidad internacional ha considerado su situación particular en las Conferencias de Almaty (2003) y Viena (2014) y sus respectivos planes de acción. Si bien los planes de acción de Almaty y Viena han significado mejoras en áreas prioritarias como tránsito, infraestructura, y cooperación e integración regional, los resultados son insuficientes. Ha habido un importante aumento de los acuerdos comerciales regionales y de bloques económicos: de un promedio de 4.4 acuerdos por país en 2017 a 9 en 2018. No obstante, tanto la tasa anual promedio del PIB como la participación de estos Estados en las exportaciones globales ha caído en el mismo período ${ }^{27}$. Además, estos mecanismos de cooperación económica para el desarrollo

\footnotetext{
${ }^{21}$ UPRETY (2006), pp. 3-6.

22 UN-OHRLLS (2019).

${ }^{23}$ FAYE et al. (2004), pp. 46-52.

${ }^{24}$ SINGER (2003), p. 32.

${ }^{25}$ COLLIER (2007), pp. 53-63; FAYE et al. (2004), pp. 49-52; UN (2015), pp. 112-114; UN-OHRLLS (2019); UPRETY (2006), pp. 130-145.

${ }^{26}$ UN (2015), pp. 104-115.

27 UN-OHRLLS (2018).
} 
tienen una desventaja, ya que profundizan la dependencia de los Estados sin litoral respecto de otros. Frente a esta situación, se ha enfatizado en la necesidad de complementar los mecanismos de las Conferencias de Almaty y Viena, buscando alternativas que les permitan promover su prosperidad de manera autónoma y menos dependiente de las decisiones de otros $^{28}$.

\subsection{Regulación de los Estados sin litoral en la CONVEMAR}

La CONVEMAR reconoce los obstáculos a los que se enfrentan los Estados sin litoral en el aprovechamiento de los océanos y establece ciertos derechos para garantizar su inclusión. La Convención entrega a estos Estados dos grupos de derechos. Unos, los que tienen que ver con el libre acceso y goce de la alta mar, y la participación en la ZEE de su región. Los segundos, los que buscan garantizar su participación equitativa en la explotación de la Zona y en la distribución de los beneficios económicos derivados de ella.

Respecto del primer grupo, el gran problema es la dificultad de ejercer los derechos. En el caso del derecho de acceso al mar (art. 125) y la libertad de navegación (art. 87), estos están condicionados a celebrar acuerdos con los Estados de tránsito. Dado que gran parte de los Estados sin litoral se encuentra en regiones conflictivas e inestables, no siempre es fácil lograr estos pactos. Las condiciones quedan libradas a los Estados de tránsito, quienes se encuentran en el lado fuerte de la negociación y pueden "tomar todas las medidas necesarias para que los Estados sin litoral no lesionen sus intereses" (art. 125).

Los derechos de aprovechamiento de los recursos en la ZEE también presentan dificultades. Los artículos 69 y 70 señalan que los Estados sin litoral y geográficamente desaventajados tienen el derecho a participar, sobre una base equitativa, en la explotación de una parte apropiada del excedente de recursos vivos de las ZEE de los Estados ribereños de la misma subregión o región, teniendo en cuenta las características económicas y geográficas pertinentes de todos los Estados interesados ${ }^{29}$. En este caso la CONVEMAR sí establece una obligación correlativa de los Estados costeros. Este derecho, no obstante, está limitado por requerimientos y condiciones adicionales: solo existe en relación con una parte apropiada del excedente de los recursos vivos, determinada por el Estado costero, con una cierta excepción para los Estados en desarrollo sin litoral, quienes tienen derecho a una "parte equitativa" en la región o subregión independientemente de si hay un excedente. Además, el ejercicio de este derecho en relación con la pesca, uno de los factores más valiosos, depende de acuerdos adicionales con los Estados costeros, quienes deben evaluar los efectos de estas actividades en las comunidades o la industria pesquera, la extensión en que los Estados sin litoral pueden ejercer su derecho y las necesidades nutricionales de los Estados. Por último, los artículos 69 y 70 pueden dar lugar a interpretaciones divergentes, dada la indeterminación de ciertos términos como "base equitativa", "excedente" o "subregión" 30.

Respecto del segundo grupo de derechos, esto es, aquellos que buscan la participación equitativa en la Zona, los problemas vinieron dados por el Al, que alteró el balance original de la Convención que buscaba favorecer tanto a los Estados en desarrollo, como a aquellos sin litoral. La Parte XI establece normas para que Estados sin litoral tengan la posibilidad de intervenir en el régimen del fondo marino, sin que su condición geográfica sea una limitante. El artículo 148 promueve la participación efectiva de los Estados en desarrollo en las actividades de la Zona, refiriéndose a sus necesidades especiales para superar los obstáculos derivados de su situación geográfica, incluyendo la lejanía de la Zona y su dificultad de acceso. Para ello, la CONVEMAR asegura su participación en los órganos deliberativos de la AIFM (art. 161).

Además, en el ejercicio de sus facultades y funciones la AIFM puede prestar atención especial a los Estados sin litoral, haciendo una excepción al mandato de no discriminación a la

${ }^{28}$ CASAL Y SELAMÉ (2015), p. 276.

${ }^{29}$ Estos derechos no se extienden, sin embargo, a la exploración y explotación de los recursos no vivos en la plataforma continental.

30 TUERK (2015), pp. 332 y 333. 
hora de otorgar concesiones para realizar actividades en la Zona establecido en el artículo 152. Para poder garantizar una participación efectiva, el artículo 266 señala que debe fomentarse la asistencia técnica en materia de ciencia y tecnología marina, particularmente dirigida a los Estados sin litoral y geográficamente desaventajados. En la misma línea se encuentran los artículos 269 y 272 en materia de cooperación técnica para la transferencia de tecnología y promoción de formas de cooperación bilateral y multilateral, los que ordenan tener especialmente en cuenta los intereses y necesidades de los Estados en desarrollo, particularmente los sin litoral y los Estados geográficamente desventajados.

Sin embargo, y a pesar de este trato favorable, la participación de los Estados en desarrollo, especialmente de aquellos sin litoral, se ha hecho muy difícil con las modificaciones incorporadas con el $\mathrm{Al}$, las que operan en sentido contrario y prevalecen sobre las normas de la Convención. Los cambios al funcionamiento de la Empresa y los límites a la transferencia de tecnología hacen casi imposible que los Estados en desarrollo sin litoral participen de la explotación de la Zona directamente.

En la siguiente sección sostengo que una forma de balancear la tensión de derechos en la CONVEMAR, teniendo en cuenta no solo su contenido formal sino también su aplicación práctica, es otorgar a los Estados en desarrollo sin litoral un lugar preferente en la distribución de los beneficios derivados de la explotación de la Zona. Esta postura se justifica tanto en las normas de la CONVEMAR que se refieren a la situación de los Estados en desarrollo y de los Estados sin litoral, como en el criterio de reparto equitativo que subyace al principio de $\mathrm{PCH}$ que gobierna la Zona. El otorgamiento de esta preferencia, es importante destacar, no implica desconocer que pueden existir otros Estados en desarrollo igualmente elegibles para un trato preferente, sino más bien reconocer la situación especial de los Estados en desarrollo sin litoral.

\section{Interpretación del principio de PCH en la CONVEMAR}

\subsection{El principio de Patrimonio Común de la Humanidad}

El artículo 140 de la CONVEMAR declara la Zona PCH y señala que los beneficios financieros y económicos derivados de ella deben repartirse de manera equitativa. Se trata de una norma guía para la interpretación y aplicación de la Parte $\mathrm{XI}^{31}$. Desde su primera incorporación en el Tratado sobre los Principios que deben regir las Actividades de los Estados en la Exploración y Utilización del Espacio Ultraterrestre, incluida la Luna y otros Cuerpos Celestes de 1967, el principio de PCH ha sido controvertido ${ }^{32}$. Esto se debe principalmente a dos grandes dificultades: por una parte, la contradicción entre las teorías legales que defienden el control privado de los recursos frente a la idea de recursos comunes; $y$, por la otra, la vaguedad del principio, que hace más difícil su aplicación práctica ${ }^{33}$. Esto ha llevado a desacuerdos entre los Estados desarrollados y en desarrollo. Los primeros han defendido una interpretación del PCH como la posibilidad de que todos hagan uso de las áreas designadas. Los segundos esperan que haya más que una igualdad formal de acceso ya que, sin la tecnología ni el capital necesarios, los Estados desarrollados se harían de casi todos los beneficios de esta explotación.

Si bien existen otros tratados internacionales que también contemplan el principio de $\mathrm{PCH}$, estos tampoco brindan una definición clara de su contenido. El principio de $\mathrm{PCH}$ fue recogido en 1970 en la Declaración de Principios que Regulan los Fondos Marinos y Oceánicos y su Subsuelo Fuera de los Límites de la Jurisdicción Nacional ${ }^{34}$. Fue insertado también en 1974 a la Declaración sobre el Establecimiento de un Nuevo Orden Económico Internacional ${ }^{35}$ y su programa de acción. Estos tratados dieron pie a nuevos instrumentos que contienen este principio como la Carta de Derechos y Deberes Económicos de los Estados de 1974 y la

\footnotetext{
31 JAECKEL et al. (2016), p. 2.

32 SHACKELFORD (2008), pp. 106-111.

33 SHACKELFORD (2008), p. 108.

34 NACIONES UNIDAS (1970).

35 NACIONES UNIDAS (1974).
} 
Declaración sobre el Derecho al Desarrollo de $1986^{36}$. Los distintos regímenes legales que contienen el principio de PCH muestran que cada uno tiene su propia versión y en ninguno su aplicación ha sido exitosa ${ }^{37}$. En todos ellos, sin embargo, la idea de equidad es central ${ }^{38}$.

A pesar de el carácter vago del principio de $\mathrm{PCH}$, la doctrina ha identificado cinco elementos constitutivos ${ }^{39}$. En primer lugar, la no apropiación, con prohibición de demandas territoriales, lo que constituye una limitación a la soberanía nacional. En segundo lugar, el manejo común de la región y los recursos a través de representantes de todas las naciones. En tercer lugar, su uso pacífico. En cuarto, la preservación para las futuras generaciones, que tiene relación con medidas de conservación y protección medioambiental. $\mathrm{Y}$, por último, la distribución equitativa de los beneficios para toda la humanidad. En el caso de la CONVEMAR, los últimos dos elementos son los más controvertidos. Aunque ambas discusiones -la conservación de la Zona y la distribución de sus beneficios- se encuentran interrelacionadas y no pueden ser resueltas independientemente, acá solo me referiré al problema del reparto equitativo.

\subsection{Interpretación del PCH y el problema del reparto equitativo}

Todo tratado requiere interpretación, especialmente cuando ha buscado compatibilizar los intereses de un gran número de Estados ${ }^{40}$. Este es el caso de la CONVEMAR, que pretende un equilibro entre los intereses contrapuestos de los Estados desarrollados y los no desarrollados respecto de la Zona ${ }^{41}$. Como señala Philip Allott, los tratados son desacuerdos puestos por escrito ${ }^{42}$; el resultado del compromiso de actores que frecuentemente difieren en sus ideas, intereses y deseos ${ }^{43}$. Por ello, si no podemos reconocer una verdad detrás de las palabras del tratado y ellas pueden significar lo que el intérprete quiere que signifiquen, es necesario tener reglas claras que no hagan de la interpretación un mero ejercicio de poder. Interpretar un tratado es similar a dictar sus términos ${ }^{44}$.

Aun cuando la importancia de las reglas de interpretación es cuestionada y algunos señalan que queda mayoritariamente entregada a la virtud del intérprete ${ }^{45}$, hoy existe consenso en cuanto a la aplicación de las reglas generales de interpretación establecidas en el artículo 31 y 32 de la Convención de Viena sobre el Derecho de los Tratados (CVDT). Así lo ha señalado la jurisprudencia de la Corte Internacional de Justicia, el Tribunal Europeo de Derechos Humanos y la Corte Interamericana de Derechos Humanos en numerosas sentencias ${ }^{46}$. Una interpretación del $\mathrm{PCH}$ a la luz de estas normas nos permite sostener, en primer término, que este principio promueve el interés de la humanidad como un todo, entendida como transespacial y transtemporal, esto es, incluyendo tanto a las generaciones presentes como a las futuras en cualquier lugar del planeta. Con ello, la humanidad pasa a ser un nuevo actor del derecho internacional cuyo interés debe ser defendido. Esto da al derecho internacional una nueva perspectiva que se aleja de aquella que regula solamente las relaciones entre Estados ${ }^{47}$.

Uno de los grandes problemas interpretativos del principio en la CONVEMAR, sin embargo, está en determinar cómo deben ser distribuidos los beneficios de la explotación de la Zona para que exista un reparto equitativo que vaya en interés de toda la humanidad (art. 140.2). Esto ha sido uno de los aspectos más debatidos de la aplicación del PCH en la

\footnotetext{
${ }^{36}$ NACIONES UNIDAS (1986)

37 SHACKELFORD (2008), p. 139.

38 BOURREL et al. (2016), p. 2.

39 SHACKELFORD (2008), p. 109.

${ }^{40}$ AUST (2005), p. 88.

${ }^{41}$ BOURREL et al. (2016), p. 2.

${ }^{42}$ ALLOT (1999), p. 43

${ }^{43}$ KLABBERS (2010), p. 26.

${ }^{44}$ KLABBERS (2010), pp. 20 y 24.

${ }^{45}$ KLABBERS (2010), pp. 35-37.

${ }^{46}$ NOVAK (2013), pp.72-74.

47 TANAKA (2015), p. 19
} 
CONVEMAR. Tanto durante las negociaciones como después de su adopción se trató de un tema divisorio, al punto que el Al debilitó las referencias relativas al reparto de beneficios para lograr la firma de los Estados desarrollados ${ }^{48}$. La regulación específica de esta distribución quedó entregada a la AIFM, encargada del manejo común de los recursos para el bien común, en un intento de la Convención de lograr una administración comunitaria amistosa y cooperativa ${ }^{49}$. Como era de esperar, sin embargo, hoy la AIFM se encuentra en la dificultad de determinar los parámetros de la distribución y enfrenta importantes problemas sobre la operacionalización del principio ${ }^{50}$.

En lo que sigue, adelanto una forma de dar contenido a este principio de acuerdo con la CVDT. Una interpretación literal (31.1 CVDT) del PCH sugiere que el reparto equitativo de beneficios va más allá de una simple distribución aritmética entre los Estados, y requiere considerar la situación desaventajada de algunos de ellos. El artículo 140 dispone que las actividades en la Zona se realizarán en beneficio de toda la humanidad, independientemente de la ubicación geográfica de los Estados, ya sean ribereños o sin litoral, y prestando especial consideración a los intereses y necesidades de los Estados en desarrollo y de los pueblos que no hayan logrado la plena autonomía. Su numeral segundo señala que debe hacerse una distribución equitativa de los beneficios, mediante el mecanismo que disponga la AIFM sobre una base no discriminatoria. Las normas generales sobre este mecanismo, de acuerdo con el artículo 160, también hacen referencia a los intereses y necesidades de los Estados en desarrollo y los pueblos que no han alcanzado su autonomía. Estos principios se encuentran en consonancia con otras normas de la CONVEMAR que, de acuerdo con el artículo 31.2 CVDT, deben ser tomadas como contexto para una interpretación sistemática. Ejemplo de ello son el artículo 143, que se refiere a la investigación científica, el 149 respecto de los objetos arqueológicos y el 150 en relación con el aprovechamiento de los recursos de la Zona, etc.

La idea de que el reparto equitativo va más allá de una igualdad formal también se desprende de una interpretación histórica (art. 32 CVDT). El discurso de Arvid Pardo ante la ONU, que sirvió como antecedente a la CONVEMAR, presenta el PCH como una respuesta al impacto de las nuevas tecnologías en el uso y explotación de los recursos marinos que podría llevar a la destrucción de los océanos, y a la creciente desigualdad entre los países. En la sesión 22 de la Asamblea General de las Naciones Unidas Pardo urgió por un régimen internacional para gobernar el fondo del mar, explotar sus recursos y distribuir los beneficios de su venta a los países pobres en el nombre de un desarrollo rápido que les permitiera "ponerse al día" con el desarrollo ${ }^{51}$.

En este mismo sentido, una interpretación teleológica (art. 31.1 CVDT) igualmente indica que el reparto equitativo de los beneficios debe favorecer a los Estados en desarrollo. El preámbulo señala que la CONVEMAR espera ser una contribución importante al mantenimiento de la paz y la justicia y al progreso para todos los pueblos (preámb.: 1). Para ello, establece un orden jurídico cuyo objetivo es, entre otros, la utilización equitativa y eficiente de los recursos (preámb.: 4) y la realización de un orden económico internacional justo y equitativo que tenga en cuenta los intereses de toda la humanidad y, en particular, los intereses y necesidades especiales de los Estados en desarrollo, sean ribereños o sin litoral (preámb.: 5). El artículo 139 de la Convención hace responsable de forma compartida a todos los Estados y organizaciones de concurrir al logro de estos objetivos.

\subsection{El desafío de un reparto equitativo}

Para darle contenido al criterio de equidad en el marco de la CONVEMAR es necesario recurrir a principios de justicia. La interpretación conforme a estos principios encuentra su

\footnotetext{
48 JAECKEL (2016), p. 2.

${ }^{49}$ SHACKELFORD (2008), p. 139.

50 JAECKEL (2016), p. 2.

51 NACIONES UNIDAS (1967).
} 
fundamento normativo en el artículo 31.3.c CVDT que permite para estos efectos recurrir a otras normas de derecho internacional. En este sentido, la Comisión de Derecho Internacional de las Naciones Unidas señaló en 2006 que el artículo 31.3.c opera como una llave maestra que, entre otras cosas, permite una interpretación integradora del orden jurídico que autoriza a recurrir a fuentes externas al tratado relevantes para su interpretación, como otros tratados, normas derivadas de la costumbre internacional o los principios generales del derecho ${ }^{52}$. Dentro de estas normas podemos acudir a los principios generales del derecho reconocidos por las naciones civilizadas los que son, de acuerdo con el artículo 38.1.c del Estatuto de la Corte Internacional de Justicia una fuente primaria del derecho internacional.

La equidad es un principio general del derecho reconocido por las naciones civilizadas ${ }^{53}$. Esta ha ido ganando reconocimiento a través del derecho internacional del medio ambiente como un medio para la protección de los derechos de las futuras generaciones ${ }^{54}$. Sin embargo, su contenido puede ser muy variado y dependerá de qué teorías de justicia se suscriban. Estas visiones pueden ser tan dispares, que algunos Estados desarrollados han argumentado que una repartición equitativa establece una proporcionalidad entre el monto de la inversión y los beneficios, mientras que un grupo Estados en desarrollo consideran que debe ser por partes iguales o en atención a las necesidades de cada Estado ${ }^{55}$.

¿Cómo entender, entonces, el reparto equitativo de los beneficios? En este trabajo defiendo una interpretación del principio de equidad que justifica una distribución de beneficios que privilegie a los Estados más desfavorecidos. Siguiendo un razonamiento basado en principios suficientarios y argumentos sobre justicia compensatoria y responsabilidad colectiva, sostengo que los Estados en desarrollo sin litoral son un grupo de Estados que tienen argumentos de equidad fuertes para recibir un tratamiento preferencial en la distribución de los beneficios. Mi posición se basa no solo en que los Estados en desarrollo sin litoral se encuentran en peores condiciones de desarrollo sostenible que los Estados en desarrollo costeros (Secciones 3.a. y 3.b.), sino que sostiene que, de acuerdo con principios de reparación, existen razones adicionales para justificar un reparto que los favorezca.

\subsection{Suficiencia, reparación y responsabilidad colectiva}

Para determinar en qué consiste un reparto equitativo de los beneficios derivados de la explotación de la Zona es importante tener a la vista la posibilidad de que operen principios compensatorios de justicia, esto es, que deba repararse a algunos Estados por injusticias pasadas. En esta sección analizaré la situación particular de los Estados en desarrollo sin litoral, sosteniendo que incluso de acuerdo con principios de justicia mínimos, como los presentados por Thomas Pogge ${ }^{56}$ y David Miller ${ }^{57}$, hay buenas razones para justificar una reparación, la que puede tener lugar por medio de la distribución de los beneficios derivados de la explotación de la Zona. En primer lugar, explicaré por qué algunas teorías de la justicia, como las recogidas por los geoístas, sugieren que la creación de la ZEE justifica compensar a los Estados en desarrollo sin litoral por el perjuicio causado. En segundo lugar, defenderé la necesidad de compensar a estos Estados fundada en el deber negativo de no dañar a otros mediante la imposición de un régimen institucional injusto, examinando la forma en que puede operar la responsabilidad colectiva en este caso, particularmente atendiendo a la regulación incorporada con el Al. Sostengo que para ello no es necesario apelar a principios igualitarios de justicia, los que por lo general son más demandantes y menos ecuménicos en el plano global, sino que bastan razones de suficiencia.

\footnotetext{
52 SANDS Y COMMISSION (2010), p. 57.

${ }^{53}$ Véase, por ejemplo, GOUGOURINIS (2009) y WHITE (2004).

${ }^{54}$ BOURREL et al. (2016), pp. 3 y 4.

${ }^{55}$ BOURREL et al. (2016), p. 3.

56 POGGE (2005) y POGGE (2011).

${ }^{57}$ MILLER (2007).
} 
Los principios igualitarios señalan que es injusto que algunas personas tengan prospectos de vida muy distintos a otras, y que por lo tanto es necesario combatir la desigualdad, entendida como la existencia de desventajas relativas ${ }^{58}$. El fundamento principal de estas teorías está en que todos los seres humanos tenemos un mismo valor moral o una igual dignidad, y que debemos ser tratados y tratarnos como iguales. En este sentido, puede entenderse como una posición que busca favorecer un mayor grado de igualdad en el ingreso o bienestar de las personas.

Los principios suficientarios, por su parte, son menos demandantes y se centran en las desventajas absolutas, considerando que existe un deber de justicia de poner a todas las personas sobre un determinado límite. Lo relevante es que la mayoría posible de seres humanos esté por sobre un determinado umbral que les asegure las condiciones mínimas para llevar una vida digna y poder perseguir sus propios planes de vida ${ }^{59}$. Si bien el límite es controvertido y se ha entendido en general como la erradicación de la pobreza extrema, esto no solo dice relación con las condiciones materiales para la subsistencia, sino que además implica la protección de otros derechos y la satisfacción de demandas de igual respeto. De acuerdo con estos principios, se considera moralmente justificada la transferencia de recursos de los que están en mejor posición a los que están peor si esto pone más gente sobre el umbral.

Existen importantes razones para centrarnos tanto en la disminución de la desigualdad como de la pobreza absoluta. Por una parte, la existencia de desigualdad extrema parece incompatible con la idea de que todas las personas tenemos igual valor moral e iguales pretensiones de tener una vida floreciente. Por la otra parte, los efectos de la desigualdad extrema también son indeseables en la medida que la concentración de poder ha hecho a nuestras instituciones más sensibles a las preferencias de los más ricos, lo que hace que los problemas globales que nos afectan a todos se hagan más difíciles de resolver, e impacten en la pervivencia de la pobreza absoluta ${ }^{60}$.

Sin embargo, a pesar de que existen buenas razones tanto para luchar contra la desigualdad como contra la pobreza, la recepción de los principios igualitarios ha sido menos extendida en el ámbito global. La resistencia en el siglo pasado -siguiendo las teorías del liberalismo igualitario rawlsiano- a no reconocer en los problemas globales obligaciones de justicia sino entenderlos como problemas de ayuda humanitaria, ha permeado hoy como una dificultad para aceptar deberes de justicia como las que se derivan de las teorías igualitaristas en el ámbito internacional ${ }^{61}$. Si bien cada vez hay mayor consenso en que las obligaciones de justicia pueden extenderse más allá de las fronteras nacionales, ya sea como consecuencia de la igual dignidad de todos o del hecho de la globalización que ha convertido en menos relevantes las fronteras, los autores generalmente tienden a reconocer deberes menos exigentes en el ámbito global que el nacional. A esto se suma que los principios igualitarios son generalmente criticados por la 'objeción de nivelar hacia abajo', que supone que la búsqueda de la igualdad puede llevar a que todos estemos igualmente mal o peor, y por los problemas de practicabilidad que suponen una imposibilidad actual de lograr estos objetivos.

Por estos motivos, y considerando que los principios suficientarios alcanzan para justificar el argumento de este trabajo, me basaré en estos, entendiendo que no obstan a la persecución de fines más demandantes como los que impone la igualdad. Como señala Paula Casal ${ }^{62}$, un enfoque suficientario negativo, esto es, aquel que considera injusto que la gente esté bajo un determinado umbral y que sostiene que debamos hacer lo posible por mejorar su posición, no obsta que las diferencias sobre ese límite sean relevantes en una aproximación a la justicia

\footnotetext{
${ }^{58}$ Para una discusión actual sobre las teorías igualitaristas a nivel global véase GILABERT (2012) y ARMSTRONG (2019).

59 Para una aproximación a las ideas suficientarias véase FRANKFURT (1987).

${ }^{60}$ ARMSTRONG (2019), pp. 37-48.

${ }^{61}$ En este sentido, el Derecho de Gentes (2001) de John RAWLS marcó un hito respecto de cómo pensar acerca de la justicia global. Para Rawls, si las sociedades tienen un set de instituciones que permiten a los ciudadanos llevar a cabo vidas 'decentes', entonces las desigualdades que existan entre ellas no generan problemas de justicia. Esta teoría ha sido, no obstante, ampliamente desafiada por autores como BEITZ (1979); POGGE (2002); BROCK (2009), entre muchos otros.

62 CASAL (2007), pp. 299-303.
} 
compatible con deberes dinámicos. Si bien la determinación del umbral es siempre un problema difícil, lo entenderé como la existencia de pobreza extrema, en consonancia con el ODS1 de los Objetivos de Desarrollo Sostenible.

Estos principios permiten desarrollar el argumento del reparto equitativo de la siguiente manera. A partir de la CONVEMAR, el mayor cambio en el derecho internacional del mar ha sido la expansión de los derechos de los Estados costeros sobre el espacio marítimo. Con la creación de la ZEE, la Convención cerró los océanos, lo que tuvo efectos profundamente desiguales. Por un lado, los grandes beneficiados fueron los "big six" (Gran Bretaña, Estados Unidos, Francia, Nueva Zelanda, Australia y Rusia), quienes alcanzaron 54 millones de kilómetros cuadrados de ZEE, 39 millones de los cuales se derivan de territorios de ultramar y protectorados ${ }^{63}$. Por el otro, los más afectados fueron los Estados sin litoral, quienes quedaron excluidos de la repartición de territorios e impedidos de acceder a importantes recursos, como la mayoría de los recursos ictícolas, los que por regla general se encuentran cerca de la costa. Estos Estados no solo quedaron relegados a derechos residuales sobre la explotación de los recursos vivos de la ZEE. Además, la configuración del nuevo orden global trajo consigo una disminución del espacio donde pueden ejercer sus derechos y participar de las prácticas que configuren nuevos derechos a través de la costumbre internacional, estableciéndose una mayor distancia con la alta mar y una mayor dependencia respecto de sus vecinos para acceder a ella.

De acuerdo con teorías geoístas, el planeta y sus recursos naturales pertenecen a todos, porque nadie ha contribuido a su creación. Esto justifica algún tipo de propiedad igualitaria, tal como fuera argumentado por John Locke o Henry George. En razón de la limitación de los recursos, la apropiación o aprovechamiento por parte de unos afecta a otros, quienes deben ser compensados producto de la exclusión. ${ }^{64}$. Si bien con la formación de los Estados la mayoría del planeta y sus recursos no fueron distribuidos de acuerdo con principios de justicia, esto no significa que no podamos evaluar la repartición resultante de acuerdo con ellos. En el caso de la ZEE, se extendió la soberanía de algunos Estados sobre territorios que antes eran comunes, atendiendo a un criterio geográfico. De acuerdo con el proviso de Locke, con esta repartición no se dejó "tanto y tan bueno" para los Estados sin litoral, quienes por tanto pueden hacer valer argumentos de equidad para exigir una compensación por su pérdida.

Por razones geopolíticas es muy difícil considerar una redistribución del territorio para esta reparación. No obstante, hay alternativas intermedias. Por ejemplo, existen propuestas que buscan enmendar injustos como el producido por la CONVEMAR a través de impuestos sobre la propiedad de los recursos naturales o la explotación de estos. Hillel Steiner ${ }^{65}$, por ejemplo, sostiene que tenemos un derecho negativo a no ser privados de los recursos naturales y a que no se limite nuestra posibilidad de usarlos, salvo que seamos compensados. Steiner plantea un "Fondo global" con un impuesto que grave el $100 \%$ del valor de mercado de los recursos naturales, que luego se reparta por el número de habitantes. Pogge ${ }^{66}$, por su parte, propone un "Dividendo de recursos globales", que grave a los Estados con una tasa fija por la explotación o consumo de los recursos naturales, que sirva para terminar con la pobreza extrema ${ }^{67}$.

En el caso de la CONVEMAR, una compensación de este tipo podría funcionar por medio del diseño de un sistema impositivo que grave la explotación de los recursos de la Zona y opere como una vía de corrección de la arbitrariedad de la repartición realizada a través de la creación de la ZEE y de las normas del Al que perjudican la participación de los Estados en desarrollo en las actividades en la Zona. Este sistema puede ser un mecanismo efectivo de redistribución, y su aceptación puede facilitarse atendiendo a distintas consideraciones. Primero, porque se espera que las riquezas del fondo marino sean tan abundantes, que impuestos de este tipo permitan combatir la pobreza severa y la desigualdad. Segundo, porque como la base del impuesto es la naturaleza, se pueden encontrar justificaciones tan distintas como las teorías de Hobbes o

${ }^{63}$ ARMSTRONG (2020), p. 10

${ }^{64}$ CASAL (2011), p. 313.

${ }^{65}$ STEINER (2011), pp. 330 y 331

${ }^{66}$ POGGE (2011), pp. 338 y 339.

67 Para una discusión acerca de los impuestos sobre los recursos naturales véase ARMSTRONG (2017), pp.177-200. 
Rawls. Y, tercero, porque un impuesto sobre los recursos naturales puede favorecer el medio ambiente y las futuras generaciones, incentivando un uso menos depredador y más responsable ${ }^{68}$.

Pogge ${ }^{69}$ plantea otra forma de entender la necesidad de compensación, complementaria del argumento anterior, basada en que la pobreza extrema viola el deber negativo de no dañar a otros, en la medida que puede ser evitada mediante un diseño distinto del orden global que sea más justo. Existe responsabilidad de los ciudadanos de los Estados más ricos junto a las élites de los Estados en desarrollo ya que son ellos quienes diseñan y mantienen este orden, aun cuando puede haber otras alternativas igualmente viables. Para que el orden global en el que vivimos sea justo, Pogge sostiene que debe cumplir con un mínimo, de modo que el orden institucional garantice que los derechos humanos se satisfagan en la medida de lo razonablemente posible. Así no vulneraremos el deber negativo de no dañar al no imponer un orden que vulnere evitablemente los derechos humanos, sin que exista una compensación por ello. En este caso, Pogge se refiere a la satisfacción de derechos humanos como la ausencia de pobreza extrema. Las obligaciones positivas nacen de la vulneración del deber negativo de no perjudicar a otros, y consisten en reformar el orden para satisfacer los derechos humanos mínimos.

En este contexto, la creación de un orden para los mares y océanos de la CONVEMAR contribuye a la situación de desventaja de los Estados en desarrollo, especialmente de aquellos sin litoral, y al déficit de los derechos humanos de sus habitantes, existiendo la posibilidad de un orden alternativo más justo, como el que se había planteado antes del Al; o uno alternativo en que se hubiera otorgado mejores derechos a los Estados en desarrollo sin litoral sobre la ZEE o se hubiera garantizado su participación en las actividades a realizarse en la Zona. Para Pogge, la necesidad de compensación surge no solo del hecho de que muchos Estados en desarrollo sin litoral tienen indicadores sociales y de desarrollo humano muy bajos, sino también de que el orden existente, en este caso la CONVEMAR y el posterior Al, los ha puesto en una situación desventajosa respecto de los Estados ribereños y los Estados desarrollados, existiendo un orden alternativo posible más favorable.

Es necesario considerar, sin embargo, que la rectificación de injusticias pasadas presenta un gran desafío, en la medida que puede afectar a quienes no han intervenido en su producción. Si bien la identificación de los responsables y la transferencia de responsabilidad a las generaciones futuras es un tema complejo que excede a este artículo, se ha sostenido que hay razones que permiten justificar medidas rectificatorias aunque afecten los planes de personas que no hayan jugado un rol importante en la generación de las condiciones, en los casos de un esquema pasado extremadamente injusto ${ }^{70}$. De acuerdo con David Miller ${ }^{71}$, por ejemplo, las razones para actuar en un caso como este no solo proceden de una responsabilidad por haber producido un determinado resultado, sino también de la responsabilidad remedial, que tiene que ver con la necesidad de mejorar circunstancias gravosas incluso en aquellos casos en que no las hemos producido. Ambas responsabilidades reflejan aspectos contrastantes de la condición humana. Por una parte, la de ser agentes, de cuya autonomía surge la obligación de responder de las ganancias y pérdidas que generamos para nosotros mismos y para otros. Por la otra, la de ser vulnerables, necesitados de otros para cumplir con nuestros objetivos. La responsabilidad remedial requiere de situaciones suficientemente graves y urgentes que necesiten ser subsanadas. Para Miller, en la misma línea de Pogge, estas pueden consistir en una distribución injusta, como el caso de la ZEE o las modificaciones introducidas por el Al, si estas contribuyen a la insatisfacción de los derechos humanos esenciales, entendidos como aquel mínimo de demandas que de manera razonable todos tenemos respecto de otros ${ }^{72}$.

\footnotetext{
${ }^{68}$ CASAL (2011), p. 312.

${ }^{69}$ POGGE (2005), p. 55.

${ }^{70}$ BUCHANAN (1975), pp. 419 y 420.

${ }^{71}$ MILLER (2007), p. 81.

${ }^{72}$ MILLER (2007), pp. 178-185
} 
Ambos autores sustentan la obligación de reparar en una distribución o un hecho dañoso que produce una insatisfacción de derechos humanos. Es decir, su posición es que debemos velar por el cumplimiento de los derechos humanos no en términos positivos, sino como aquellos mínimos que necesitamos para poder tener una vida digna. Se trata de derechos humanos como estándares morales y no como normas jurídicas. Para estos autores, el fundamento de la responsabilidad remedial estaría en principios de justicia suficientarios, esto es, aquellos que sostienen que lo relevante es que la mayoría posible de seres humanos esté por sobre un determinado umbral que les asegure las condiciones mínimas para llevar una vida digna y poder perseguir sus propios planes de vida $^{73}$. En este sentido, se justificaría una compensación a los Estados en desarrollo sin litoral por la exclusión en la ZEE y la creación de un orden injusto, aun cuando afecte a personas que no han participado en la producción de este resultado.

Si bien es difícil determinar el umbral sin caer en la arbitrariedad, la ausencia de pobreza extrema parece un mínimo razonable. Esto permite justificar que la distribución de los beneficios favorezca a los Estados sin litoral en desarrollo, quienes no han podido alcanzar este mínimo (como sostuve en la sección 3), en la medida que podría contribuir a la posibilidad de estos de avanzar hacia un desarrollo sostenible, de acuerdo con lo establecido en los ODS. Una distribución que tome especialmente en cuenta los intereses y necesidades de los Estados sin litoral en desarrollo no solo contribuiría al ODS 1 "eliminar la pobreza en todas sus formas" y ODS10 "disminuir la pobreza dentro y entre los países", sino que además podría servir para el cumplimiento del ODS 8 "promover el crecimiento económico sostenido, inclusivo y sostenible, el empleo pleno y productivo y el trabajo decente para todos", el ODS 9 "industria, innovación e infraestructura" y el ODS 12 "garantizar modalidades de consumo y producción sostenibles". Todo ello, teniendo a la vista los ODS 13 "adoptar medidas urgentes para combatir el cambio climático y sus efectos" y ODS 14 "conservar y utilizar en forma sostenibles los océanos, los mares y los recursos marinos para el desarrollo sostenible", que son los que mayores desafíos impondrán al desarrollo de estas nuevas actividades. Esto resulta particularmente importante considerando la naturaleza interdependiente de los objetivos, los que deben ser interpretados armónicamente, entendiendo que una de las contribuciones más interesantes de los ODS es la importante conexión entre justicia oceánica y otras metas de la justicia global ${ }^{74}$.

\section{Conclusión.}

La próxima explotación de la Zona traerá importantes retos para el derecho internacional del mar. Con el inicio de la minería submarina se espera que se obtengan grandes beneficios económicos derivados de la extracción de nódulos polimetálicos, sulfitos polimetálicos y corteza de ferromanganeso, los que podrán ser explotados por Estados o particulares con patrocinio de Estados por medio de una licencia de la AIFM. Probablemente, estas actividades serán realizadas por aquellos que hoy cuentan con la tecnología y el capital para hacerlo. La Convención señala, sin embargo, que esta explotación debe ir en interés de toda la humanidad, conforme al principio de $\mathrm{PCH}$, y que debe existir un reparto equitativo de los beneficios económicos y financieros derivados de ella. Este tema ha sido controvertido desde las negociaciones de la CONVEMAR, con visiones contrapuestas de los Estados desarrollados y en desarrollo, con y sin litoral. Uno de los grandes desafíos a los que nos veremos enfrentados, entonces, será determinar en qué consiste ese reparto equitativo para que efectivamente se beneficie a la humanidad como un todo.

En este trabajo he sostenido una interpretación del reparto equitativo que se aleja de una igualdad formal, y que favorece a los Estados en desarrollo, particularmente los sin litoral. He basado esta interpretación en principios suficientarios de justicia y en argumentos sobre justicia compensatoria y responsabilidad colectiva. Para ello, he analizado las consecuencias distributivas de la creación de la ZEE y la Zona, y los efectos que tuvo para los Estados en

${ }^{73}$ FRANKFURT (1987).
${ }^{74}$ ARMSTRONG (2020). 
desarrollo la modificación de la regulación de la Zona con el Al. Además, he dado cuenta de las especiales dificultades para alcanzar un desarrollo sostenible a las que se ven enfrentados los Estados sin litoral, y cómo las respuestas entregadas por el derecho internacional, incluida la CONVEMAR, han resultado insuficientes.

Por último, he interpretado el principio de patrimonio común de la humanidad y el mandato de reparto equitativo a la luz de las normas de la CVDT, incorporando la equidad como un principio general del derecho. He concluido que hay razones para dar un lugar preferente en el reparto a los Estados en desarrollo sin litoral fundadas en la obligación de compensar por su exclusión en la repartición de la ZEE, la necesidad de reparación por la imposición de un orden institucional previsiblemente injusto que da lugar a pobreza extrema, y las obligaciones derivadas de la responsabilidad remedial en los casos de situaciones extremadamente injustas.

\section{BIBLIOGRAFÍA CITADA}

ALLOTT, PHILIP (1999): “The Concept of International Law", en: European Journal of International Law (Vol. 10), pp. 31-50.

ANDERSON, DAVID (1995): "Resolution Agreement Relating to the Implementation of Part XI of the UN Convention on the Law of the Sea: A General Assessment", en: International Comparative Law Quarterly (Vol. 44), pp. 313-326.

ARMSTRONG, Chris (2017): Justice and Natural Resources (Oxford, Oxford University Press).

ARMstrong, Chris (2019): Why Global Justice Matters (Cambridge, Polity Press).

ARMStrong, Chris (2020): "Ocean Justice: SDG 14 and Beyond", en: Forthcoming in Journal of Global Ethics. Disponible en: https://papers.ssrn.com/sol3/papers.cfm?abstract_id=3611553 [visitado el 13 de octubre de 2020].

AUST, ANTHONY (2005): Handbook of International Law (Cambridge, Cambridge University Press).

BeItZ, ChARLes (1979): Political Theory and International Relations (Princeton, Princeton University Press).

Bourrel, Marie; Thiele, Torsten y Duncan, Currie (2016): "The common heritage of mankind as a means to assess and advance equity in deep sea mining", en: Marine Policy (Vol. 95). Disponible en: https://www.Ise.ac.uk/iga/assets/documents/publications/2017/common-of-heritage-ofmankind-deep-sea-mining.pdf [visitado el 14 de octubre de 2020].

BROCK, GILLIAN (2009): Global Justice: a Cosmopolitan Account (Oxford, Oxford University Press).

Buchanan, Allen (1975): "Distributive Justice and Legitimate Expectations", en: Philosophical Studies (Vol. 28, № 6), pp. 419-425.

BUGA, IRINA (2015): "Between Stability and Change in the Law of the Sea Convention: Subsequent Practice, Treaty Modification, and Regime Interaction", en: Rothwell, Donald; Oude Elfernik, Alex; Scott, Karen y Stephens, Tim (Eds.), The Oxford Handbook of the Law of the Sea (Oxford, Oxford University Press), pp. 46-68.

CASAL, Paula (2007): “Why Sufficiency is Not Enough”, en: Ethics (Vol. 117, №2), pp. 296-326.

CASAL, PAULA (2011): "Global Taxes on Natural Resources", en: Journal of Moral Philosophy (Vol. 8, № 3), pp. 307-327.

Casal, Paula y Selamé, Nicole (2015): "Sea for the Landlocked: a new Sustainable Development Goal?", en: Journal of Global Ethics (Vol. 11, № 3), pp. 270-279.

CHURCHILL, RobIN (2015): The 1982 United Nations Convention on the Law of the Sea, en: Rothwell, Donald; Oude Elfernik, Alex; Scott, Karen y Stephens, Tim (Eds.), The Oxford Handbook of the Law of the Sea (Oxford, Oxford University Press), pp. 24-45. 
Collier, PAul (2007): The Bottom Billion (Oxford, Oxford University Press).

Faye, Michael; Mcarthur, John; Sachs, JefFrey y Snow, Thomas (2004): "The Challenges Facing Landlocked Developing Countries", en: Journal of Human Development (Vol. 5, № 1). pp. 31-68.

FrankfurT, HARRY (1987): “Equality as a Moral Ideal,” en: Ethics (Vol. 98, № 1), pp. 21-43.

GILABERT, PABLo (2012): From Global Poverty to Global Equality (Oxford, Oxford University Press).

GOURGOURINIS, ANASTASIOS (2009): “Delineating the Normativity of Equity in International Law”, en: International Community Law Review (Vol. 11), pp. 327-347.

JaECKEL, Aline; ARDRON, JefF y GJeRde, KRISTINA (2016): "Sharing benefits of the common heritage of mankind - Is the deep seabed mining regime ready?" en: Marine Policy (Vol. 70). Disponible en: http://dx.doi.org/10.1016/j.marpol.2016.03.009 [visitado el 14 de octubre de 2020].

KLABBERS, JAN (2010): “Virtuous Interpretation”, en: Fitzmaurice, Malgosia; Merkouris, Panos y Elias, Olufemi (Eds.), Treaty Interpretation and the Vienna Convention on the Law of Treaties: 30 Years on (Leiden-Boston, Martinus Nijhoff Publishers), vol. 1, pp. 17-38.

LODGE, Michael (2015): "The Deep Seabed", en: Rothwell, Donald; Oude Elfernik, Alex; Scott, Karen y Stephens, Tim (Eds.), The Oxford Handbook of the Law of the Sea (Oxford, Oxford University Press), pp. 226-253.

MILLER, DAVID (2007): National Responsibility and Global Justice (Oxford, Oxford University Press).

NACIONES UNIDAS, ASAMBLEA General (1967): "Examination of the question of the reservation exclusively for peaceful purposes of the seabed and the ocean floor, and the subsoil thereof, underlying the high seas beyond the limits of present national jurisdiction, and the use of their resources in the interests of mankind. General debate, AG 2340 (XXII) (1 de noviembre de 1967)". Disponible en: https://www.dipublico.org/conferencias/mar/pdffiles/papers/2340.pdf [visitado el 13 de octubre de 2020].

NACIONeS UnidAs, Asamblea General (1970): "Declaración de Principios que Regulan los Fondos Marinos y Oceánicos y su Subsuelo Fuera de los Límites de la Jurisdicción Nacional, AG 2749 (XXV) (17 de septiembre de 1970)". Disponible en: https://www.dipublico.org/conferencias/mar/pdffiles/papers/2750.pdf [visitado el 13 de octubre de 2020].

NACIONES UnidAS, AsAmblea General (1974): "Declaración sobre el Establecimiento de un Nuevo Orden Económico Internacional, AG 3201 (S-VI) (1 de mayo de 1974)". Disponible en: http://www.gloobal.net/iepala/gloobal/fichas/ficha.php?entidad=Textos\&id=3331 [visitado el 13 de octubre de 2020].

NACIONES UNIDAS, ASAMBLEA GeneRAL (1986): “Declaración sobre el Derecho al Desarrollo, AG 41/128 (4 de diciembre de 1986)". Disponible en: https://www.ohchr.org/sp/professionalinterest/pages/righttodevelopment.aspx [visitado el 13 de octubre de 2020].

NovAK, FABIÁN (2013): "Los criterios para la interpretación de los tratados", en: Themis Revista de Derecho (№ 63), pp. 71-88.

Pogge, Thomas (2002): World Poverty and Human Rights (Cambridge, Polity Press).

PogGe, Thomas (2005): "Severe Poverty as a Violation of Negative Duties", en: Ethics and International Affairs (Vol. 19, № 1), pp. 54-83.

Pogge, THOMAS (2011): "Allowing the Poor to Share the Earth", en: Journal of Moral Philosophy (Vol. 8, № 3), pp. 335-352.

RaWLS, John (2001): The Law of Peoples: With the The Idea of Public Reason Revisited (Cambridge, Harvard University Press). 
SANDS, PHILIPPE y COMISSIONS, JeFFery (2010): "Treaty, Custom and Time: Interpretation/Application?", en: Fitzmaurice, Malgosia; Merkouris, Panos y Elias, Olufemi (Eds.), Treaty Interpretation and the Vienna Convention on the Law of Treaties: 30 Years on, (LeidenBoston, Martinus Nijhoff Publishers), vol. 1, pp. 39-58.

SHACKELFORD, SCOTT J. (2008): "The Tragedy of the Common Heritage of Mankind", en: Stanford Environmental Law Journal (Vol. 27), pp. 101-157. Disponible en: https://ssrn.com/abstract=1407332 [visitado el 13 de octubre de 2020].

Singer, Peter (2003): Un solo mundo: la ética de la globalización (Traducc. Francisco Herreros Vázquez, Barcelona, Paidós).

Steiner, Hillel (2011): “The Global Fund: A Reply to Casal”, en: Journal of Moral Philosophy (Vol. 8, № 3), pp. 328-334.

TANAKA, YOSHIFUMI (2015): The International Law of the Sea (Cambridge, Cambridge University Press).

TUerk, Helmut (2015): "Landlocked and Geographicaly Disadvantaged States", en: Rothwell, Donald; Oude Elfernik, Alex; Scott, Karen y Stephens, Tim (Eds.), The Oxford Handbook of the Law of the Sea (Oxford, Oxford University Press), pp. 325-345.

UN (2015): "Global Sustainable Development Report.". Disponible en: https://sustainabledevelopment.un.org/content/documents/1758GSDR\%202015\%20Advance\%2 OUnedited\%20Version.pdf. [visitado el 29 de enero de 2020].

United Nations Development Programme (UNDP) (2019): "Human Development Report 2019. Beyond income, beyond averages, beyond today: Inequalities in human development in the $21^{\text {st }}$ century". Disponible en: http://hdr.undp.org/sites/default/files/hdr2019.pdf [visitado el 29 de enero de 2020].

UN - OHRLLS (United Nations Office of the High Representative fOR the LeAst DeVeloped Countries, Landlocked Developing Countries and SMall Island DeVeloping Countries) (2018): "Landlocked Developing Countries Factsheet". Disponible en: http://unohrlls.org/customcontent/uploads/2018/10/LLDC-fact-sheet.pdf. [visitado el 29 de enero de 2020].

UN - OHRLLS (2019): "Landlocked Developing Countries Fact Sheet 2019. Midterm Review of the Vienna Programme of Action". Disponible en: http://unohrlls.org/customcontent/uploads/2020/01/Factsheet_2019.pdf. [visitado el 29 de enero de 2020].

UPRETY, KISHOR (2006): The Transit Regime for Landlocked States. International Law and Development Perspectives (Washington D.C., The World Bank).

White, MARgaret (2004): “Equity: A General Principle of Law Recognised by Civilised Nations", en: Queensland University of Technology Law and Justice Journal (Vol. 4, № 1), pp. 103-116.

NORMAS JURÍDICAS CITADAS

ACUERDO DE IMPLEMENTACIÓN (Al). Nueva York, 7 de agosto de 1994.

CARTA DE DERECHOS Y DEBERES ECONÓMICOS DE LOS ESTADOS. Nueva York, 14 de noviembre de 1974.

CONVENCIÓN DE LAS NACIONES UNIDAS SOBRE EL DERECHO DEL MAR (CONVEMAR). Jamaica, 10 de diciembre de 1982.

ConVención de Viena sobre deReCho de los tratados (CVDT). Viena, 23 de mayo de 1969.

Estatuto de la CoRTe INTERnacional de Justicia (CIJ). San Francisco, 18 de abril de 1956. 
TRATADO SOBRE LOS PRINCIPIOS QUE DEBEN REGIR LAS ACTIVIDADES DE LOS ESTADOS EN LA EXPLORACIÓN Y UTILIZACIÓN DEL ESPACIO ULTRATERRESTRE, INCLUIDA LA LUNA Y OTROS CUERPOS CELESTES. Nueva York, 27 de octubre de 1967. 